\title{
EDITORIAL
}

\section{Neuroethics: An Introduction}

This section of the current issue of Neurotherapeutics will focus on neuroethics. The preceding section, on neuroimaging, with its focus on elucidating brain function, is a good stepping-off point for discussions of moral reasoning, choice, and free will, in that these are frequently considered to be in the forefront as such imaging research proceeds. Furthermore, many diseases of the central nervous system either directly alter aspects of brain function that impair reasoning or affect aspects of cognition that directly or indirectly influence aspects of human functioning that are at the core of who we are as persons and personalities. As researchers in neuroscience apply brain imaging to investigate issues such as morality and choice—and as therapies such as deep brain stimulation are developed that not only alter one's physical well-being by ameliorating tremor but also have the potential to induce changes in the person's emotional life- researchers in bioethics have begun to address the ethical issues raised by these developing technologies and therapies as well as by parallel advances in our understanding of how the brain works. The issues of interest to bioethicists range from the philosophical, such as constructs of free will, discussed by Bok in this issue, to issues that are very concrete, such as the question of invasive placebo controls raised by Horng and Miller.

The relevance of neuroethics to clinical care, research, and our everyday lives positions the articles in the neuroethics section of this issue to be of interest to clinicians, clinical researchers, basic scientists, patients with brain disease, their caregivers, and their loved ones.

Hilary Bok begins with a theoretical paper that raises a basic question about free will, determinism, and choice. Is it inevitable, she asks, that advances in our understanding of the neuroscience underlying choice will lead to the abandonment of these constructs, which have been staples of Western thought since the Enlightenment? Such challenges are not new, of course, and were raised by logical-positivist philosophers almost 100 years ago, as well as by the behaviorist B.F. Skinner in his 1971 book, Beyond Freedom and Dignity. Bok's challenge to the claim that neuroscience will vanquish free will is clear and incisive, and deserves to be debated widely.

On the other end of the spectrum in neuroethics-and notably underrepresented in the literature-is the concrete reality of those living daily with neurological disease and disability and their interactions with the biomedical community. Cohen et al. discuss the differences in perspective among scientists, clinicians, industry sponsors, and those living with Parkinson's disease and how those differences translate into ethical conflicts. They propose a patient-centered paradigm for clinical research and medical care to help resolve some of these conflicts and to facilitate the development of new therapies.

In addition to general questions about the ethical foundations of thought and reasoning, medical advances raise specific ethical and clinical challenges. One potential advance, the early detection of cerebral palsy by new applications of brain electrical signal mapping, is challenged by John Freeman. Freeman attacks the scientific basis of the proposed technique and questions the validity of claims that it indeed can do what adherents and promoters claim. At first blush, this might seem to be an argument over scientific interpretation rather than the application of ethical reasoning, but Freeman demonstrates how learning from prior errors alerts one to the vulnerability of the medical and legal professions to claims of truth when the evidence (as here, in his opinion) does not support the claim. Freeman's article demonstrates that moral reasoning does not exist in a vacuum; rather, medical ethics must combine both an understanding of the medical and epidemiologic issues as well as a good grasp of the moral implications of the decision under consideration.

Two articles in this volume address specific ethical issues that deserve scrutiny: the role of and justifications for the use of placebo-controlled trials in evaluating treatments for progressive debilitating diseases (Horng and Miller) and the challenges clinicians face in caring for adults with dementia, particularly the tensions among abstract ethical principles, the best interests of the ill person, and the concerns of the family (Blass).

Lux raises similar issues in relationship to individuals who have experienced a traumatic brain injury-that is, how binding should a person's opinions and requests be when autonomy may be limited by an injured brain. The challenge in persons with traumatic brain injury is that many of their cognitive capacities are not impaired and they may appear quite intact. Both Blass and Lux note the importance of involving individuals who have a knowledge of and close relationship with the person prior to the disease or injury, and suggest that these prior relationships help identify and justify a substituted judgment involvement. 
Finally, Whitehouse and Waller present a critique of the field of neuroethics and call for consideration of the view that the act of labeling disorders-and academic fields- has ethical implications. Although they examine the constructs of Alzheimer's disease and involuntary emotional expression disorder (IEED) as "conditions," and the field of neuroethics as an exemplar of a discipline whose proponents have shaped its discourse in a particular social and political direction, they do not consider that all categories are influenced by the values of the era in which they are created and are influenced by social as well as nonsocial factors. An example of such a socialpolitical belief is the view that constructs such as free will and choice will disappear when their neuroanatomy, neurophysiology, and molecular biology are "understood."

This special section does not cover all of the important issues that fall under the rubric of neuroethics, but it does illustrate the importance of the issues that it considers, as well as the rigor that the discipline can bring to such discussions. We suggest that the discipline of neuroethics will best meet its goals by including a wide range of topics related to brain, behavior, emotional life, moral life, and the diseases that afflict the central nervous system-and by including not only the voices of scholars, but also those of patients.

Debra J. H. Mathews, Ph.D., M.A. Assistant Director for Science Programs Johns Hopkins Berman Institute of Bioethics Johns Hopkins University Baltimore, MD

Peter Rabins, M.D., M.P.H. Professor

Departments of Psychiatry and Behavioral Sciences Johns Hopkins Berman Institute of Bioethics Johns Hopkins University Baltimore, MD 